\title{
População urbana exposta aos riscos de deslizamentos, inundações e enxurradas no Brasil
}

\author{
Urban population exposed to risks of landslides, floods and flash \\ floods in Brazil
}

\author{
Silvia Midori Saito ${ }^{1}$ \\ Mariane Carvalho de Assis Dias ${ }^{2}$ \\ Regina Célia dos Santos Alvalá ${ }^{3}$ \\ Claudio Stenner 4 \\ Cayo de Oliveira Franco 5 \\ Julia Vicente Martins Ribeiro 6 \\ Pilar Amadeu de Souza 7 \\ Rodrigo Amorim Souza de Moraes Santana ${ }^{8}$
}

\begin{abstract}
Resumo
O conhecimento sobre a população urbana expostos ao risco de desastres é imprescindível para o planejamento de ações de prevenção e resposta. O objetivo do presente trabalho é analisar as condições de exposição de grupos populacionais em áreas de risco de desastres em 479 municípios brasileiros. O procedimento metodológico baseou-se na associação de dados censitários e de áreas de risco de deslizamentos, enxurradas e inundações. A base de dados foi constituída de dados sociodemográficos e ambientais, que permitiu caracterizar os moradores e as moradias em áreas de risco. Os resultados evidenciaram que o maior total de pessoas expostas estava na região Sudeste do país, embora, em termos proporcionais, na região
\end{abstract}

\footnotetext{
${ }^{1}$ Centro Nacional de Monitoramento e Alertas de Desastres Naturais, São José dos Campos, SP, Brasil.silvia.saito@cemaden.gov.br

${ }^{2}$ Centro Nacional de Monitoramento e Alertas de Desastres Naturais, São José dos Campos, SP, Brasil.mariane.assis@cemaden.gov.br

${ }^{3}$ Centro Nacional de Monitoramento e Alertas de Desastres Naturais, São José dos Campos, SP, Brasil.regina.alvala@cemaden.gov.br

4 Instituto Brasileiro de Geografia e Estatística. Rio de Janeiro, Rio de Janeiro, Brasil. claudio.stenner@ibge.gov.br

5 Instituto Brasileiro de Geografia e Estatística. Rio de Janeiro, RJ, Brasil. cayo.franco@ibge.gov.br

6 Instituto Brasileiro de Geografia e Estatística. Rio de Janeiro, Rio de Janeiro, Brasil. julia.ribeiro.cemaden@ibge.gov.br

7 Instituto Brasileiro de Geografia e Estatística. Rio de Janeiro, Rio de Janeiro, Brasil. pilar.souza.cemaden@ibge.gov.br

8 Instituto Brasileiro de Geografia e Estatística. Rio de Janeiro, Rio de Janeiro, Brasil. rodrigo.cemaden@ibge.gov.br

Recebido em: 11/12/2018. Aceito para publicação em: 19/06/2019.
} 
Nordeste 15 a cada 100 pessoas viviam em áreas de risco. Estes dados permitem selecionar áreas que demandam ações prioritárias, auxiliando na implementação de políticas públicas regionais que promovam a redução de danos humanos no país.

Palavras-chave: população em áreas de risco, dados censitários, exposição a desastres

\begin{abstract}
The knowledge about the urban population exposed to disaster risk is essential for planning prevention and response actions. The aim of this study was to analyze the exposure conditions of the population groups at disaster risk areas in 479 Brazilian municipalities. The methodological procedure was based on the association of census data and landslides, flash floods, and floods risk areas. The database was made up of sociodemographic and environmental data, which allowed the characterization of the residents and the houses at-risk areas. The results showed that the largest number of people exposed was in the Southeast region of the country, although, in proportional terms, in the Northeast region 15 out of every 100 people lived in risk areas. These data could allow the selection of areas for priority actions, in order to help the implementation of regional public policies that promote the reduction of human losses in Brazil.
\end{abstract}

Keywords: at-risk population, census data, disaster exposure.

\title{
Introdução
}

No Brasil, avanços têm sido dispendidos na última década para a identificação da vulnerabilidade a desastres no contexto de mudanças ambientais (DEBORTOLI et al.; 2015), para o desenvolvimento de indicadores de risco de desastres (Almeida et al.; 2016) e de vulnerabilidade social a ameaças naturais (HUMMELL et al.; 2016), considerando todo o país. Outras iniciativas têm se dedicado às análises regionais (MENEZES et al.; 2018; RONCANCIO; NARDOCCI, 2016), seja para o desenvolvimento de novas metodologias ou para aplicação daquelas já existentes (ALVES 2006; DESCHAMPS, 2006; ALMEIDA, 2010; GOERL et al. 2012; ASSIS DIAS et al.; 2017). Todos os trabalhos mencionados têm em comum o uso de dados do Instituto Brasileiro de Geografia e Estatística (IBGE), estes restritos aos setores censitários do censo demográfico, realizado a cada 10 anos no país. Portanto, análises mais detalhadas, em escala intraurbana, ficam limitadas 
aos recortes desses setores, não havendo a possibilidade de redimensionálos, com o risco de super ou subestimar os dados.

No intuito de suprir a limitação acima destacada, alguns trabalhos sobre vulnerabilidade tem realizado o levantamento detalhado de dados, por meio de entrevistas. Contudo, para fins de análise em escala nacional, tal método torna-se restritivo, especialmente em razão do alto custo demandado para a produção de dados em escala mais detalhada. Logo, os dados do censo demográfico acabam se constituindo como os únicos a subsidiarem análises de vulnerabilidade. Por outro lado, a base de dados do censo demográfico do IBGE origina-se de informações mais detalhadas, denominada face de quadra, que se constitui em uma unidade menor ao setor censitário. Esse dado não é disponibilizado publicamente, a fim de preservar o sigilo dos informantes. Entretanto, esses dados são de alta relevância para a caracterização da população e das moradias expostas ao risco de desastres. Assim sendo, no presente trabalho visou-se analisar as condições dos grupos populacionais expostos em áreas de risco de desastres em 479 municípios críticos, distribuídos nas diferentes regiões do Brasil, a partir dos dados obtidos com a metodologia proposta pelo IBGE e Cemaden (2018). Na análise, mais detalhada, buscou-se distinguir as características desses grupos, bem como de suas moradias, o que constituiu em avanço significativo para o melhor conhecimento do risco de desastres no país

Ainda que seja notória a alta dinâmica de ocupação em áreas de risco de desastres no Brasil, e que os dados utilizados neste estudo são os do Censo 2010, considera-se que a contribuição desta análise possibilitou explorar o potencial da base de dados para caracterização dos grupos populacionais expostos em áreas de risco. Por se tratar de uma base de dados inédita, nota-se que os resultados apresentados podem evidenciar realidades até então pouco conhecidas.

Em termos práticos, uma das grandes vantagens desse tipo de metodologia é a padronização do procedimento em escala nacional. Assim, a 
presente análise pode subsidiar ações de monitoramento e emissão de alertas, a exemplo do que é desenvolvido pelo Centro Nacional de Monitoramento e Alertas de Desastres Naturais (Cemaden), unidade de pesquisa do Ministério de Ciência, Tecnologia, Inovações e Comunicações. Em 2012, no escopo do Plano Nacional de Gestão de Riscos e Respostas a Desastres, 821 municípios prioritários foram selecionados para serem monitorados por esse Centro, por apresentarem histórico de ocorrências de desastres relacionados a movimentos de massa (deslizamentos, corridas de massa, solapamentos de margens, terras caídas, queda/rolamento de blocos rochosos e processos erosivos) e/ou decorrentes de processos hidrológicos (inundações, enxurradas, alagamentos). Ressalta-se que alguns municípios que não foram considerados como prioritários em 2012 também passaram a ser monitorados pelo Cemaden, uma vez que dispunham de mapeamento de áreas de risco de movimentos de massa e/ou de processos hidrológicos (IBGE; CEMADEN, 2018). O monitoramento é realizado por especialistas em Hidrologia, Desastres, Meteorologia e Geodinâmica, que acompanham a atuação de sistemas meteorológicos e condições hidrológicas e geodinâmicas, bem como de possíveis impactos socioambientais causados por desastres (CEMADEN, 2019).

\section{Riscos de desastres relacionados a deslizamentos, enxurradas e inundações no contexto urbano brasileiro}

No Brasil, a (re)ocorrência de registro de impactos socioeconômicos causados por desastres de origem hidrometeorológica evidencia a necessidade da inclusão inadiável dessa pauta na agenda de políticas públicas. A prevenção de desastres exige uma atuação conjunta de ações, especialmente relacionadas a proteção civil, habitação, infraestrutura, educação e saúde. Ainda que esforços nessa direção tenham sido notados, especialmente na última década a partir do lançamento do Plano Nacional 
de Gestão de Risco e Resposta a Desastres (COSTA; PIMENTEL, 2017), infelizmente não foram suficientes para uma efetiva redução de desastres no país.

Muito embora processos geológicos e hidrológicos façam parte da dinâmica natural da superfície terrestre, a combinação com a intensificação da ocupação urbana em áreas suscetíveis, i.e. planícies de inundação e encostas declivosas, resulta em sistemas vulneráveis a deslizamentos e inundações. Caso o sistema social esteja preparado para enfrentamento desses processos, serão registrados reduzidos impactos sociais, econômicos e ambientais. Por outro lado, sistemas sociais despreparados, mesmo diante de processos de baixa magnitude, são incapazes de lidar com os seus efeitos.

Os dois cenários acima explicitados se associam a conceitos relevantes para a presente discussão. Os processos naturais, ou induzidos pela ação antrópica, com potencial de afetarem um determinado sistema social, provocando perdas de vidas, impactos socioeconômicos ou degradação ambiental, são conhecidos como ameaças (UNISDR, 2017). De acordo com a Codificação Brasileira de Desastres (BRASIL, 2017), os desastres estão relacionados a duas categorias: i) natural, a qual se subdivide em geológico, hidrológico, meteorológico, climatológico e biológico, e todos os seus respectivos subgrupos; ii) tecnológico, que podem estar relacionados a substâncias radioativas, produtos perigosos, incêndios urbanos, obras civis, transporte de passageiros e cargas não perigosas.

O sistema social, por sua vez, é um retrato das condições determinadas por fatores físicos, sociais, econômicos ou ambientais, que aumentam a suscetibilidade de um individuo, comunidade ou sistema de ser afetado pelas ameaças, o que vem a caracterizar o conceito de vulnerabilidade. (UNISDR, 2017). Logo, o risco de desastre está relacionado com o potencial de perda de vidas, prejuízos ou ativos afetados que podem ocorrer a um sistema, determinado em função da ameaça, exposição, vulnerabilidade e capacidade (UNISDR, 2017). 
No Brasil, o número de pessoas vulneráveis tem aumentado, dada a falta de planejamento de uso e ocupação do solo (MARICATO; OGURA; COMARU, 2010). Além disso, pode-se atribuir a outros fatores, como a estrutura do mercado imobiliário e a ausência histórica do Estado na efetivação de políticas habitacionais no país, restringindo acesso a locais seguros para moradia (CARDOSO, 2006). Diante de um país cada vez mais urbano e da impossibilidade de resolver o problema de moradia a curto prazo, a tendência de aumento de pessoas expostas em áreas de risco de desastres é uma realidade para se conviver. Ainda que não seja possível prever todas as ameaças, as consequências são previsíveis e podem ser minimizadas através da redução de vulnerabilidade (GONÇALVES, 2012).

Para atenuar os prejuízos causados por desastres, torna-se imprescindível conhecer as ameaças e as vulnerabilidades, uma vez que a partir da identificação dos indivíduos e comunidades expostas é que se pode definir medidas estruturais e não-estruturais. Além disso, pode-se melhor compreender como uma mesma ameaça produz diferentes graus de impactos para os sistemas sociais. Nesse sentido, dados da exposição ao risco (e.g. do ambiente construído) e dados socioeconômicos e demográficos podem apoiar no conhecimento das características da população, que aumentam ou diminuem a sua capacidade de preparação para resposta e recuperação de um desastre (CUTTER, 2011).

Os estudos sobre vulnerabilidade da população a desastres tem avançado paulatinamente no Brasil, em especial nos últimos dez anos. Do ponto de vista teórico, em muitos deles têm sido destacados a necessidade de se entender a vulnerabilidade resultante de processos de desigualdades (CARMO, 2014), como um conceito multidimensional (MARANDOLA; D'ANTONA, 2014), de sua relação com o desenvolvimento (AVILA; MATTEDI, 2017), e com as causas principais e pressões dinâmicas (MARCHEZINI; WISNER, 2017). A aplicação para diversos contextos que tangenciam a temática de desastres tambémtem abordado a vulnerabilidade 
escolar (MARCHEZINI; TRAJBER; MUÑOZ, 2018); inserção em sistemas de alerta de desastres (SAITO, 2018); e na saúde (LONDE et al, 2018).

$\mathrm{Na}$ gestão pública, por outro lado, o avanço é ainda um pouco mais lento. No âmbito do Plano Nacional de Gestão de Risco e Resposta a Desastres do governo federal (2012 a 2015), destacam-se duas ações. A primeira trata da setorização de áreas de risco de deslizamentos e inundações, conduzida pelo Serviço Geológico do Brasil, no período de 2011 a 2014, que possibilitou a estimativa de habitações e moradores expostos em 821 municípios brasileiros (CPRM, 2019). Tal setorização foi utilizada para fins de monitoramento e emissão de alertas, para ações de prevenção e resposta bem como para obras de contenção (SAMPAIO et al, 2015). A segunda ação refere-se ao projeto "Dados e Análise da Vulnerabilidade a Desastres Naturais para Elaboração de Mapas de Risco e Apresentação de Proposta de Intervenções para Prevenção de Desastres", sob responsabilidade da Secretaria Nacional de Proteção e Defesa Civil. A perspectiva era de realizar o levantamento de vulnerabilidade de 275 municípios brasileiros afetados por desastres (SCHADECK et al, 2015). Contudo, a falta de aderência entre as metodologias aplicadas impossibilitou a aplicação dos resultados em ações de prevenção.

\section{Material e Métodos}

No presente artigo foram analisados dados relativos a 479 municípios distribuídos nas diferentes regiões do Brasil, integrantes da lista de 821 municípios críticos para ações nacionais de gestão de risco e resposta a desastres naturais (Figura 1). 
Figura 1: Municípios analisados por região do Brasil.

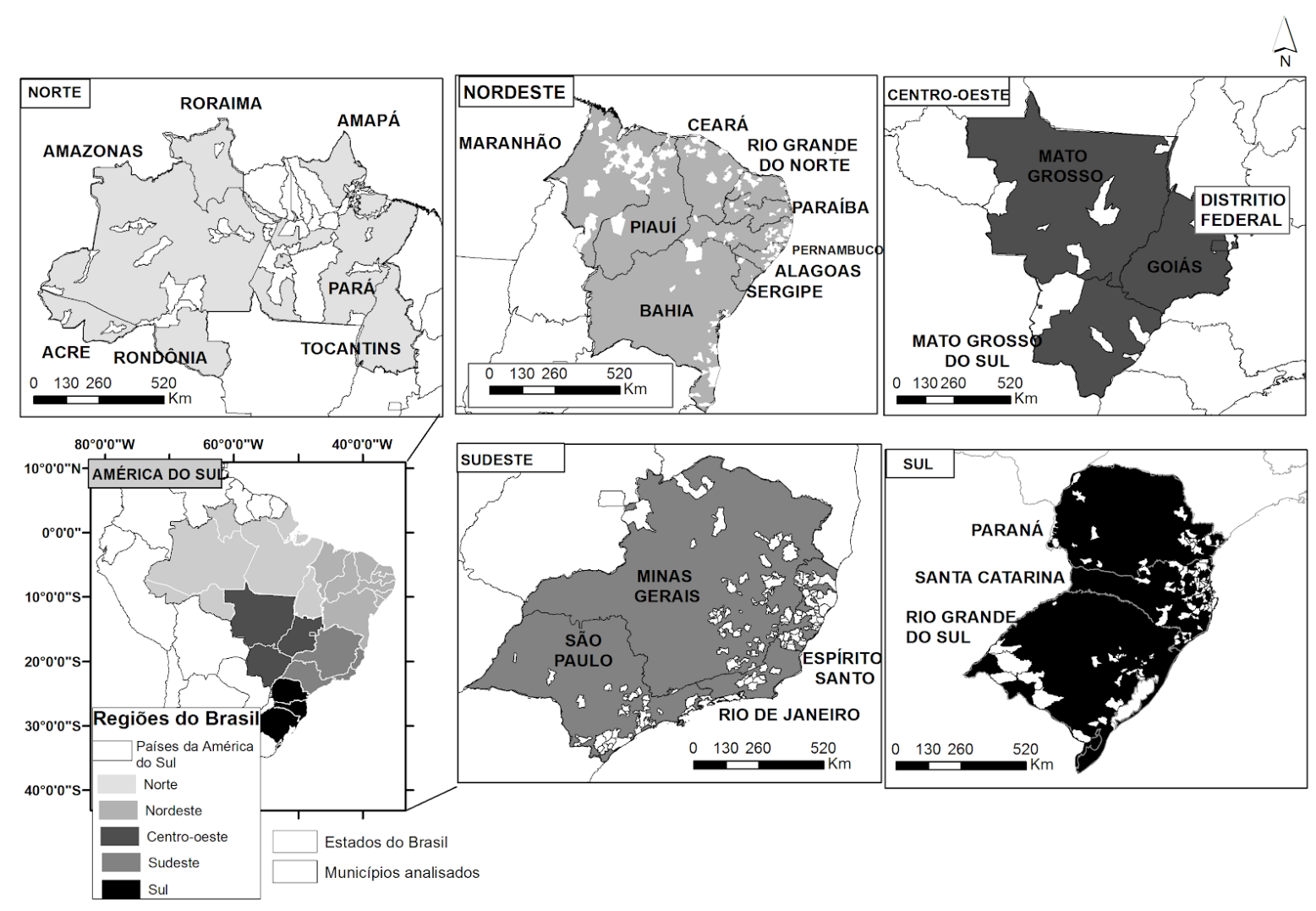

Os procedimentos metodológicos adotados no presente trabalho consistem na compatibilização das bases de dados do censo demográfico com mapeamentos de áreas de risco de deslizamentos, inundações e enxurradas para a caracterização dos grupos populacionais expostos em áreas de risco do Brasil, a qual está detalhada em IBGE e Cemaden (2018) e em Assis Dias et al. (2018).

Os dados do censo demográfico 2010 constituem-se em uma das bases de dados utilizadas na metodologia supracitada. No Brasil, desde 1890 o censo vem sendo realizado a cada dez anos, salvo nos anos 1910 e 1930, quando foram suspensos por motivos de ordem política, e em 1990, quando foi adiado para 1991 (IBGE, 2016). A periodicidade decadal é recomendada pelo Departamento de Economia e Questões Sociais, das Nações Unidas, de modo a permitir a comparação das informações visando descrever o presente e prever o futuro (UN, 2015). Ainda que no contexto da temática de risco de 
desastres o ideal fosse o levantamento de dados com maior regularidade, o alto custo do censo demográfico é fator limitante para sua realização (UN, 2006). Para fins de análise demográfica, entende-se que o intervalo de dez anos representa um retrato da realidade de uma determinada área.

Ressalta-se que a associação entre as duas bases de dados supracitadas não pode ser feita de forma direta e automática, uma vez que elas apresentam características e aplicações distintas; logo, não há correspondência espacial entre elas. Por este motivo, o desafio metodológico constituiu-se em compatibilizar dados de geometrias completamente distintas. Para a associação dos dados censitários às áreas de risco fez-se necessário a criação de um novo recorte territorial visando compatibilizar as duas geometrias, o qual foi denominado BATER Base Territorial Estatística de Áreas de Risco. Este recorte constituiu-se em uma generalização territorial, uma vez que não é possível fazer uma interseção exata entre os dois recortes originais. Portanto, os dados gerados através das BATERs não representam quantitativamente os moradores residentes em áreas de risco, mas sim uma estimativa daqueles que vivem nelas.

A delimitação gráfica da BATER foi realizada por interpretação visual considerando o contexto espacial das áreas de risco e dos limites censitários. Para o contexto espacial das áreas de risco considerou-se os padrões de densidade e de construção das moradias inseridas nessas áreas. Para esta delimitação foram consideradas oito premissas explicitadas em IBGE e CEMADEN (2018):

a) A BATER resulta da menor área possível, produto da interseção da área de risco e as feições censitárias.

b) b) cada feição censitária e cada área de risco pertence apenas a uma BATER.

c) áreas de risco em aglomerados subnormais foram generalizadas,

d) a BATER também foi generalizada em caso da face de quadra apresentar padrão de construção e densidade semelhantes.e) a BATER 
abrangeu duas ou mais áreas de risco, diante de similaridade de padrão de densidade e construção.

f) para BATER associadas ao risco hidrológico foram consideradas faces de quadra dos dois lados da rua, pois em caso de inundação é muito provável que ambas vias sejam afetadas.

g) para BATER associadas ao risco de deslizamento, foram consideradas a forma do relevo, direcionamento das vertenes e o grau de declividade.

h) considerando que os mapas de áreas de risco eram provenientes de diferentes instituições e elaborados a partir de diferentes metodologias, os graus de risco não foram considerados,

No universo de mais de 600 variáveis da base tabular disponibilizadas pelo censo, IBGE e Cemaden (2018) selecionaram 183 para caracterizar os moradores, tais como idade, gênero, alfabetização, entre outros; e 135 para caracterizar os domicílios, como por exemplo, acesso a serviços básicos como energia elétrica, esgotamento sanitário, abastecimento de água e coleta de lixo (Tabela 1). As variáveis foram também utilizadas para caracterizar os municípios, permitindo análises comparativas entre a situação dos grupos populacionais residentes nas áreas de risco e a situação da população total residente no município. 
Tabela 1: Variáveis selecionadas para caracterização dos domicílios e da população exposta aos riscos de deslizamentos, enxurradas e inundações no Brasil.

\begin{tabular}{|c|c|}
\hline Variáveis sobre moradores & Variáveis sobre domicílios \\
\hline \multicolumn{2}{|c|}{ Número domicílios e moradores totais } \\
\hline Moradores por faixa etária & Número de domicílios não ocupados \\
\hline Moradores por gênero & $\begin{array}{l}\text { Número de domicílios por formas de } \\
\text { abastecimento de água }\end{array}$ \\
\hline Moradores por alfabetização & $\begin{array}{l}\text { Número de domicílios por formas de } \\
\text { esgotamento sanitário }\end{array}$ \\
\hline Moradores por rendimento & $\begin{array}{l}\text { Número de domicílios por formas de coleta } \\
\text { de lixo }\end{array}$ \\
\hline $\begin{array}{l}\text { Moradores por responsável do domicílio, } \\
\text { segundo gênero, alfabetização e rendimento }\end{array}$ & $\begin{array}{l}\text { Número de domicílios por formas de } \\
\text { energia }\end{array}$ \\
\hline $\begin{array}{l}\text { Moradores por condição do domicílio: } \\
\text { formas de abastecimento de água }\end{array}$ & $\begin{array}{l}\text { Número de domicílios por renda domiciliar } \\
\text { per capita, responsável e gênero }\end{array}$ \\
\hline $\begin{array}{l}\text { Moradores por condição do domicílio: } \\
\text { formas de esgotamento sanitário }\end{array}$ & $\begin{array}{l}\text { Número de domicílios por quantidade de } \\
\text { moradores }\end{array}$ \\
\hline $\begin{array}{l}\text { Moradores por condição do domicílio: } \\
\text { formas de coleta de lixo }\end{array}$ & $\begin{array}{l}\text { Número de domicílios por tipo, ex.: casa, } \\
\text { apartamento }\end{array}$ \\
\hline $\begin{array}{l}\text { Moradores por condição do domicílio: } \\
\text { formas de energia }\end{array}$ & $\begin{array}{l}\text { Número de domicílios próprios, alugados } \\
\text { ou cedidos }\end{array}$ \\
\hline
\end{tabular}

\section{Resultados e Discussões}

Na Tabela 2 são apresentadas informações gerais sobre os municípios brasileiros divididos pelas grandes regiões e dos 479 municípios analisados no presente trabalho.

Considerando o número total de municípios analisados, 29.535 áreas de risco de deslizamentos e de inundações foram mapeadas. A partir da distribuição espacial das áreas de risco e dos limites censitários foram criados 4.683 polígonos de BATER (Tabela 3). A maioria dos dados associados foi proveniente de faces de quadra (85\% dos polígonos de BATER), enquanto os demais 15\% foram originários de setores censitários. 
Tabela 2. Informações gerais sobre os municípios brasileiros e municípios analisados.

\begin{tabular}{|c|c|c|c|c|c|c|}
\hline \multirow[b]{2}{*}{ egião } & \multicolumn{3}{|c|}{ Dados Demográficos do Brasil } & \multicolumn{3}{|c|}{ Dados dos 479 municípios analisados } \\
\hline & $\begin{array}{l}\mathrm{N}^{o} \text { total de } \\
\text { municípios* }\end{array}$ & $\begin{array}{c}\text { Total de } \\
\text { habitantes* }\end{array}$ & $\begin{array}{c}\text { Densidade } \\
\text { demográfica* }\end{array}$ & $\begin{array}{c}\mathrm{N}^{\mathrm{o}} \text { de } \\
\text { municípios } \\
\text { prioritários }\end{array}$ & $\begin{array}{c}\mathrm{N}^{\mathrm{o}} \text { de } \\
\text { municípios } \\
\text { prioritários }\end{array}$ & $\begin{array}{c}\text { Total de hab. } \\
\text { nos } \\
\text { municípios }\end{array}$ \\
\hline Nordeste & 1.794 & 53.081 .950 & 34.15 & 277 & 174 & 18.663 .593 \\
\hline Sudeste & 1.668 & 80.364 .410 & 86.92 & 292 & 156 & 35.702 .009 \\
\hline Sul & 1.188 & 27.386 .891 & 48.58 & 142 & 100 & 8.429 .702 \\
\hline Norte & 449 & 15.864 .454 & 4.12 & 81 & 37 & 5.777 .132 \\
\hline Centro- & 466 & 14.058.094 & 8.75 & 29 & 12 & 1.427 .203 \\
\hline BRASIL & 5.565 & 190.755 .799 & 22.43 & 821 & 479 & 69.999 .639 \\
\hline
\end{tabular}

*Censo demográfico 2010.

Fonte: IBGE (2010), IBGE e Cemaden (2018)

Conforme destacado em Assis Dias et al. (2018) e IBGE e CEMADEN (2018), a informação por face de quadra contém o maior detalhamento possível no nível censitário, ou seja, o nível de desagregação do dado é o que mais se aproxima do tamanho e distribuição das áreas de risco no território. Isto representa um ganho de informação, uma vez que, até o presente momento, os dados censitários utilizados para associação com áreas de risco eram unicamente provenientes da informação agregada por município ou por setores censitários.

Tabela 3: Número de áreas de risco e polígonos de BATER criados por região.

\begin{tabular}{cccc}
\hline Regiões do & Número de áreas & Extensão territorial das & Número de polígonos \\
\hline Sudeste & 16.024 & 803 & 2.597 \\
Nordeste & 5.532 & 520 & 1.071 \\
Sul & 6.789 & 749 & 764 \\
Norte & 1.149 & 268 & 223 \\
Centro-Oeste & 41 & 4 & 28 \\
\hline TOTAL & 29.535 & 2.344 & 4.683 \\
\hline
\end{tabular}

Fonte: IBGE e Cemaden (2018) 
Na Figura 2 apresenta-se a estimativa do número de moradores e de domicílios expostos nas áreas de risco, por grande região.

Figura 2: Estimativa de moradores e de domicílios expostos em 479 municípios, por grande região

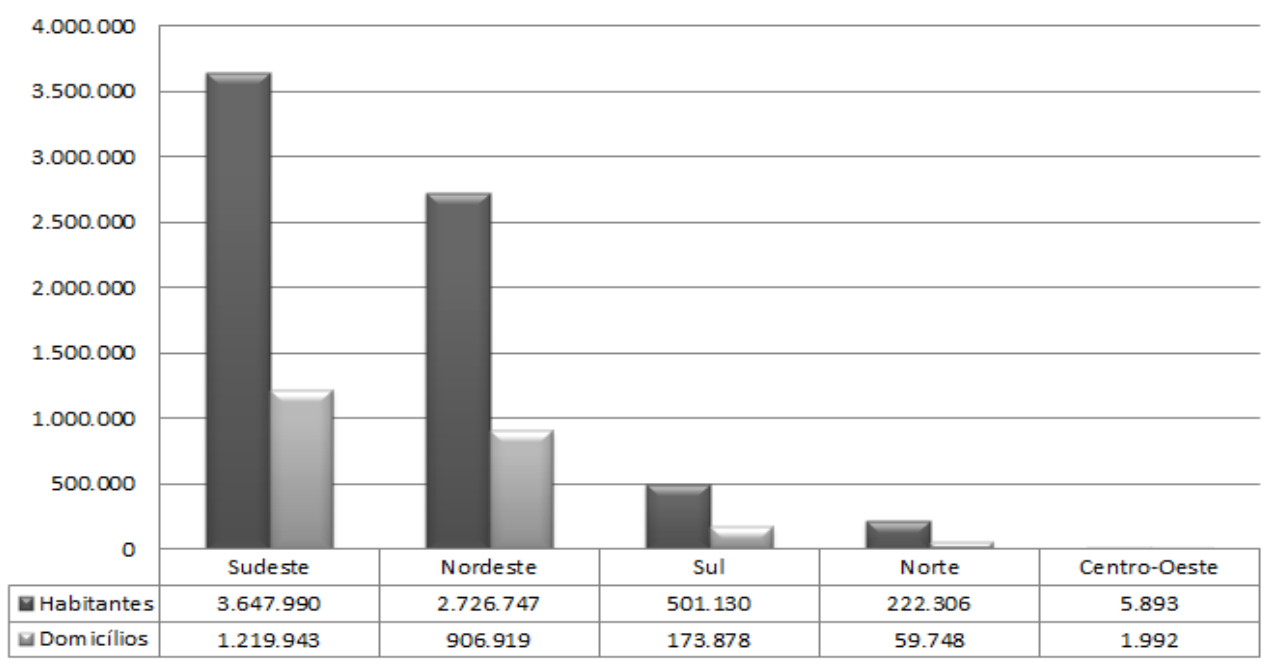

Fonte: elaboração própria

A região Sudeste concentrou a maior quantidade de áreas de risco mapeadas do país, bem como de polígonos de BATER criados. Em contrapartida, as áreas de risco da região são de pequena extensão (0,05 $\mathrm{km}^{2}$, em média) e estão localizadas próximas uma das outras.

$\mathrm{Na}$ região Nordeste, estima-se que mais de 2,7 milhões de pessoas estavam expostas ao risco, embora essa região contenha menos de $65 \%$ das áreas de risco se comparada com a região Sudeste. Associado a isto, as áreas de risco na região Nordeste têm maior extensão territorial (média de 0,09 $\mathrm{km}^{2}$ ), o que sugere que nesta região há uma maior concentração de pessoas nas áreas de risco.

A região Norte contém a maior extensão territorial das áreas de risco (média de 0,23 $\mathrm{km}^{2}$ ), em razão da maioria destas estarem em municípios localizados em grandes bacias amazônicas e, portanto, estão associadas com inundações. 
Em termos gerais, estima-se que $10 \%$ da população residente nos 479 municípios analisados estava exposta ao risco de deslizamentos e/ou inundações e/ou enxurradas, totalizando 7.104.066 pessoas em 2.131.160 moradias. A população exposta concentrou-se na faixa leste do Brasil. Nas mesorregiões (Metropolitana de Salvador, de São Paulo, de Belo Horizonte, de Recife, do Rio de Janeiro, de Fortaleza e central do Espírito Santo, Zona da Mata Mineira, Vale do Itajaí, Leste Alagoano e Leste Potiguar) agrupam mais de 76\% da população exposta estimada nos 479 municípios analisados.

Quanto às características demográficas dos municípios analisados, não se observou diferença significativa na distribuição por gênero, ou seja, aproximadamente $52 \%$ da população exposta era composta por mulheres e $48 \%$ por homens, dos quais 56\% deles eram os responsáveis pelo domicílio; no entanto, no que concerne à alfabetização, entre os $10 \%$ dos responsáveis sem alfabetização, a maioria deles (54\%) eram mulheres. Wamsler et al. (2012) destacaram o papel crucial do nível de educação da mulher como determinante para a situação de risco em que elas estão expostas, ou seja, mulheres com maior nível de escolaridade podem ter maior renda e melhor capacidade de adaptação ao risco. Em adição, a capacidade de resposta e de proteção dos grupos sociais é afetada pelo seu nível de renda, conforme destacado por Torres (2000) e por Marandola e Hogan (2005). Neumayer e Plümper (2007), analisando o impacto dos desastres naturais na expectativa de vida de homens e mulheres, apontaram que o maior impacto ocorre no sexo feminino, principalmente nas mulheres em situação de baixa renda.

A partir dos resultados obtidos, verifica-se que dos moradores expostos, em termos gerais cerca de 43\% deles viviam em domicílios sem renda ou com renda per capita de até meio salário mínimo (cujo valor era de $\mathrm{R} \$ 510,00$ em julho de 2010, aproximadamente US\$280,00). Entre as regiões do Brasil, destacam-se o Norte e o Nordeste, cujo índice de pessoas vivendo com renda per capita de até meio salário mínimo era da ordem de $62 \%$ e $55 \%$, respectivamente. Nas regiões Sul e Sudeste, os índices de pessoas com 
renda de até meio salário mínimo eram respectivamente de $21 \%$ e $36 \%$. Por outro lado, na totalidade dos municípios analisados apenas 1,6\% dos moradores residiam em domicílios com renda per capita acima de 5 salários mínimos, considerada o estrato da população com melhor situação econômica.

Entre as faixas etárias, crianças e idosos são apontadas como aquelas pessoas mais vulneráveis a desastres, por necessitarem de cuidados especiais, considerando a sua maior dependência para locomoção autônoma e menor capacidade de resistir a possíveis ferimentos (WISNER et al.; 2003). Considerando as diferentes formas de enfrentamento de cada grupo etário, Liu et al.; (2002) discutem que em contraste com a maioria dos adultos, as crianças não seriam capazes de reagir adequadamente diante da materialização do risco. Da mesma forma, as pessoas idosas tendem a apresentar maior dificuldade em locomoção autônoma, necessitando de ajuda. Além disso, esses grupos etários tendem a permanecer mais tempo em seus domicílios e, assim, podem estar mais expostos ao risco. Para a definição de idade de crianças mais vulneráveis, considerou-se aquelas menores de 5 anos, uma vez que até o ano de 2013 a obrigatoriedade do ingresso à educação infantil era aos 6 anos de idade, de acordo com a Lei 12796, de 2013 (BRASIL, 2013). Consideram-se como idosos aqueles indivíduos com idade superior a 60 anos, em conformidade com o estabelecido no Estatuto do Idoso (Brasil, 2003). Nas áreas de risco dos municípios analisados, estima-se que 17\% da população exposta pertencia ao grupo etário mais vulnerável, composto por crianças (9\%) e idosos (8\%).

Diante de um cenário de desastre espera-se responsabilidades diferenciadas entre homens e mulheres, crianças, idosos e adultos e conhecêlos pode ajudar, por exemplo, na elaboração dos planos de contingência de desastres (MAZURANA et al.; 2013). A classificação por faixa etária pode auxiliar na definição de áreas prioritárias para ação da defesa civil no momento da iminência do desastre. Por exemplo, a quantificação do número 
de pessoas expostas segundo sexo e faixa etária poderá ajudar na definição de ações para evacuação. Localidades com a maior concentração crianças e idosos, dada a maior vulnerabilidade desses grupos, merecem atenção especial e maior prontidão na retirada dessas pessoas. A população exposta entre as faixas etárias de 6 a 14 anos e de 15 a 59 anos representou o total de $16 \%$ e $66,4 \%$, respectivamente.

Do total de 7.104.066 moradores expostos nos 479 municípios, a maioria deles (75\%) residia em domicílios próprios ou quitados e do tipo casa (90\%). Em relação à situação do domicílio destaca-se as condições de esgotamento, em que $24 \%$ dos domicílios apresentaram esgotamento sanitário inadequado, ou seja, não dispunham de esgotamento por rede coletora ou fossa séptica. No contexto de riscos de deslizamentos, por exemplo, é iminente a atenção aos domicílios com outras formas de esgotamento que não sejam por meio da rede coletora ou fossa séptica, pois revelam a precariedade das condições dos domicílios. Além disso, pode ajudar a compreender melhor as intervenções humanas que favorecem a ocorrência de deslizamentos e, por sua vez, aumentam a exposição da população. A relação entre esgotamento sanitário inadequado e a presença de fossas rudimentares em áreas de risco de deslizamentos foi abordada por diversos autores como um dos condicionantes antrópicos que favorecem a ocorrência de desastres (ALHEIROS, 2003; CARVALHO et al.; 2007; ARMESTO, 2012; MIRANDOLA e MACEDO, 2014).

Diante do contexto supracitado, a situação é mais preocupante nas regiões Norte, onde $67 \%$ dos domicílios das áreas de risco tinham esgotamento sanitário inadequado, seguidas das regiões Centro-Oeste, com $54 \%$ dos domicílios e Nordeste, com 33\%. O índice nas regiões Sul e Sudeste foi de $18 \%$ e $16 \%$, respectivamente.

A Sinopse do Censo Demográfico (IBGE, 2010) aponta que no Brasil, em média, $17 \%$ dos domicílios não tinham abastecimento de água por rede geral. Nas áreas de risco dos municípios analisados, em geral o 
abastecimento de água apresenta boa cobertura, observando-se que apenas $6 \%$ dos domicílios não dispunham de abastecimento por rede geral. Destacase apenas a região Norte, onde $28 \%$ dos domicílios não tinham abastecimento por rede geral e cerca de 11\% dos domicílios em risco nessa região não tinham coleta de lixo.

\section{Caracterização dos grupos populacionais expostos por região do Brasil}

Do montante da população residente no Norte do Brasil, estima-se que $4 \%$ das pessoas viviam expostas ao risco, totalizando 222.306 pessoas em 59.748 domicílios distribuídas em 37 municípios analisados. Entre as capitais dos estados do Norte, foram analisadas Belém, PA; Boa Vista, RR; Manaus, AM; Porto Velho, RO e Rio Branco, AC. Destaca-se que do total de BATER criadas, 51\% dos polígonos gerados estão associados com áreas de riscos nessas capitais, onde estima-se que 107.796 pessoas estavam expostas em 27.861 domicílios, distribuídas ao longo das 861 áreas de risco mapeadas nessas capitais. Ou seja, 48,5\% da população estimada em risco da região Norte vivia nessas capitais.

Uma das características significativas observadas a partir dos resultados da região Norte foi a presença majoritária de crianças (13\%), seguido de idosos (6\%). Nas demais regiões, a distribuição mostra-se equilibrada, em torno de 9\% em cada faixa etária. Esse cenário pode estar relacionado com o maior número de filhos por família e a menor expectativa de vida nas localidades dessa região. Outro ponto relevante é o grande número de moradores expostos que viviam em domicílios com renda per capita de até meio salário mínimo, cujo total foi de 138.211 pessoas (62\%). Considerando o total populacional dos 37 municípios analisados, esse percentual foi de 57\%. Assim, ao se comparar com a situação dos municípios, pode-se indicar que a população exposta nas áreas de risco vivia em piores condições econômicas. 
Os domicílios nas áreas de risco da região Norte tinham as piores condições de acesso a serviços básicos, cerca de 60\% dos domicílios dispunham de esgotamento sanitário inadequado, enquanto na totalidade dos municípios esse valor foi de 44\%. Estima-se que 15.061 domicílios (25\%) não tinham abastecimento de água por rede geral e $21 \%$ não tinham medidor de energia, o que sugere a existência de ligação irregular nestas áreas. Essas características indicam a precariedade dos domicílios da região e a alta exposição da população em áreas de riscos de desastres. Considerando que as áreas de risco da região são majoritariamente de inundação nas grandes bacias amazônicas e a situação de ausência de esgotamento, infere-se maior possibilidade de contato com resíduos sólidos e consequente impacto negativo na saúde das pessoas. Além disso, ligações irregulares de energia e outras formas de abastecimento de água são mais propícias a sofrer com a sazonalidade e o impacto de eventos adversos, uma vez que pode ser interrompida a energia e o acesso à água.

A região Nordeste destacou-se por concentrar aproximadamente 15\% da população dos municípios analisados expostos em áreas de risco, com um total de 2.726.747 pessoas. No contexto das regiões metropolitanas, estimouse que mais de 1,2 milhões e 631.000 pessoas estavam expostas nas RM de Salvador e Recife, respectivamente. Estes totais referem-se apenas a 4 dos 13 municípios da RM de Salvador e a 11 dos 15 municípios da RM de Recife. Quanto às condições dos domicílios, 33\% deles dispunham de esgotamento sanitário inadequado. Este percentual é superior à média encontrada nos domicílios em risco dos 479 municípios analisados, que indicava que aproximadamente $24 \%$ dos domicílios expostos não tinham esgotamento sanitário adequado.

Outro aspecto que merece atenção refere-se às condições econômicas dos grupos populacionais expostos na região Nordeste, uma vez que $55 \%$ dos moradores expostos viviam em domicílios com renda per capita de até meio salário mínimo. Este percentual é superior àquele encontrado para os 479 
municípios analisados, ou seja, 43\% da população vivia nessa condição econômica.

Ressalta-se que esses dois indicadores apontam as situações mais críticas de exposição a desastres na região Nordeste. Estima-se que 854.012 moradores, ou seja, $21 \%$ da população exposta na região Nordeste vivia nessas condições mais críticas, com esgotamento sanitário inadequado e renda domiciliar per capita até meio salário mínimo. Destaca-se que os 32 municípios analisados estão inseridos nas regiões metropolitanas de Recife, Fortaleza, Salvador e Leste Alagoano, que apresentaram 63\% da população nesta condição.

A região Sudeste concentrou o maior número de pessoas expostas ao risco no Brasil, com estimativa de 3.647 .990 pessoas vivendo em 16.024 áreas de risco mapeadas ao longo de 156 municípios críticos. Nesta região, o principal dado de destaque relaciona-se com a situação econômica dos grupos populacionais residentes nas áreas de risco, em que 36\% das pessoas expostas vivia em domicílios com renda per capita até meio salário mínimo. Ao observar a totalidade dos municípios analisados, identificou-se que $20 \%$ da população vivia nessa condição, evidenciando que nas áreas de risco da região Sudeste havia a maior proporção do estrato mais pobre da população.

Destaca-se também a concentração de $71 \%$ da população exposta na região Sudeste vivendo em 38 municípios das regiões metropolitanas de São Paulo, Rio de Janeiro e Belo Horizonte, totalizando 2.583.705 pessoas. A distribuição nas faixas etárias mais vulneráveis mostrou-se equilibrada, sendo $9,6 \%$ de crianças e 8,6\% de idosos. Quanto às condições dos domicílios, $16 \%$ deles dispunham de esgotamento sanitário inadequado e 6\% sem medidor.

$\mathrm{Na}$ região Sul estima-se que 501.130 pessoas e 173.878 domicílios estavam expostos ao risco, estes distribuídos em 6.789 áreas de risco mapeadas. A concentração de crianças e idosos apresentava-se em torno de 9\%. Mais da metade da população exposta na região Sul (282.893 
moradores) concentrou-se em 55 municípios distribuídos pelo Vale do Itajaí (24), Grande Florianópolis (12), região Metropolitana de Curitiba (11) e região Metropolitana de Porto Alegre (8).

Destaca-se, no estado de Santa Catarina, o Vale do Itajaí, onde estão 83\% (5.650) das áreas de risco mapeadas na região Sul e cuja estimativa era de 153.616 moradores expostos. Isto indica que 14\% da população moradora nos 24 municípios críticos do Vale do Itajaí viviam em áreas de risco. Esta região destaca-se também pela alta suscetibilidade à ocorrência de desastres naturais e recorrência de eventos de deslizamentos e inundações, inclusive de grande porte. Estes eventos impactam diversos municípios situados nas bacias do rio Itajaí e Itapocu, o que tem causado frequentemente danos e prejuízos elevados (JACOBI, MOMM-SCHULT e BOHN, 2013).

A região Centro-Oeste conta com 41 áreas de risco mapeadas em 12 municípios críticos, caracterizadas por pequenos polígonos relacionados aos risco de inundação, de deslizamentos e de processos erosivos, os quais não estão associadas com eventos de grande magnitude.

A partir dos resultados obtidos para essa região constatou-se que 5.893 pessoas e 1.992 domicílios estavam expostos aos riscos. Destacam-se os municípios de Corumbá e Campo Grande, ambos no estado do Mato Grosso do Sul, com 44\% da população exposta.

Nas áreas de risco do Centro-Oeste havia maior número de pessoas vivendo em domicílios com até meio salário mínimo (36,5\%) em comparação com a totalidade dos municípios (23\%). Aproximadamente 52\% dos domicílios das áreas de risco dispunham de esgotamento sanitário inadequado, índice muito superior às demais áreas dos 479 municípios analisados, que é de $17 \%$ dos domicílios nessa condição. 


\section{Conclusões}

No presente trabalho, a partir da avaliação usando dados em escala intraurbana de 479 municípios, foi possível conhecer as condições sociais e econômicas dos grupos populacionais expostos em áreas de risco de desastres no Brasil. Os dados evidenciaram duas situações críticas e específicas dos moradores expostos: a pior situação econômica das famílias e a maior concentração de domicílios com esgotamento sanitário inadequado se comparados com as situações dos municípios como um todo. Nos municípios analisados, $27 \%$ dos moradores viviam em domicílios com renda per capita de até meio salário mínimo; contudo, ao analisar especificamente os moradores expostos em áreas de risco, esse percentual alcançou $43 \%$. Ao analisar a variável esgotamento sanitário, os resultados também revelaram situação similar, isto é, enquanto $17 \%$ dos domicílios dos 479 municípios avaliados têm esgotamento sanitário inadequado, especificamente nas áreas de risco este valor alcançou $24 \%$ dos domicílios.

A análise por regiões brasileiras evidenciou cenários de exposição específicos. Se, por um lado, a região Sudeste concentrou o maior número de pessoas expostas, por outro lado, na região Nordeste a cada 100 pessoas residentes da região, 15 viviam expostas ao risco de deslizamentos ou inundações. Na região Sudeste identificou-se que os 38 municípios analisados das regiões metropolitanas de São Paulo, do Rio de Janeiro e de Belo Horizonte concentraram $71 \%$ da população exposta da região. $\mathrm{Na}$ região Sul, constatou-se que mais de $50 \%$ da população em risco também vivia nas grandes sub-regiões, como no Vale do Itajaí, na Grande Florianópolis, e nas regiões Metropolitanas de Curitiba e de Porto Alegre.

A metodologia desenvolvida pelo IBGE e Cemaden (2018) evidencia o potencial de se utilizar os dados do Censo Demográfico para fins de prevenção de desastres. Diversas aplicações podem ser realizadas nas 
demais etapas de gestão de risco de desastres, auxiliando na redução de danos humanos. Destaca-se, ainda, a possibilidade de se replicar a metodologia a partir de novos censos demográficos, permitindo um acompanhamento temporal dos dados. Tais aspectos reforçam a necessidade de levantamento contínuo dos censos, para viabilizar a implementação de políticas públicas em redução de risco de desastres no país.

Os resultados possibilitaram identificar áreas que demandam ações prioritárias nos âmbitos social e econômico, relevantes para auxiliar na implementação de políticas públicas regionais que promovam a redução de danos humanos no país. Adicionalmente, os resultados ainda permitem evidenciar que para a redução do risco de desastres no Brasil, torna-se necessário a articulação com outras políticas públicas, relacionadas, por exemplo, com habitação, saneamento e educação.

\section{Agradecimentos}

Os autores agradecem ao Conselho Nacional de Desenvolvimento Científico e Tecnológico (CNPq) pelo apoio financeiro para a segunda autora e ao INCT Climate Change Projeto Fase 2 (Bolsa CNPq 465501 / 2014-1 / Chamada Pública MCTI / CNPQ / CAPES / FAPESP N ${ }^{\circ}$ 16/2014). Este artigo é uma contribuição da Rede Brasileira de Pesquisa sobre Mudanças Climáticas Globais FINEP / Rede CLIMA 01.13.0353-00

\section{Referências Bibliográficas}

ALHeiros, M. M.; SOUZA, M. A. A.; BITOUN, J.; MEDEIROS, S. M. G. M.; AMORIM JÚNIOR, W. M. Manual de ocupação dos morros da região metropolitana do Recife.. Programa Viva o Morro, $1^{\mathrm{a}}$ ed. FIDEM, Recife, 360p, 2003.

ALMEIDA, L. Q. Vulnerabilidade social aos perigos ambientais. Revista da ANPEGE, 6(06), 151-176, 2010. https://doi.org/10.5418/RA2010.0606.0010

ALMEIDA, L. Q.; WELLE, T.; BIRKMANN, J. Disaster risk indicators in Brazil: A proposal based on the world risk index. International Journal of Disaster Risk Reduction, 17, 251-272. 2016. https://doi.org/10.1016/j.ijdrr.2016.04.007

ALVES, H. P. D. F. Vulnerabilidade socioambiental na metrópole paulistana: uma análise sociodemográfica das situações de sobreposição espacial de problemas e riscos sociais e 
ambientais. Revista Brasileira de Estudos de População, 23 (1), 43-59, 2006. https://doi.org/10.1590/S0102-30982006000100004

ARMESTO, R. C. G. Caderno 4: ação da água da chuva no planeta Terra. Parte 2. 2012. Disponível em: <http://www.cprm.gov.br/publique/media/cadernoIV_17_09_2012.pdf $>$. Acesso: 12 de de 2015.

ASSIS DIAS, M. C.; SAITO, S. M.; FONSECA, M. R. S. Aplicação de dados censitários para caracterização da população exposta em áreas de risco de deslizamentos em Blumenau, Santa Catarina. Revista Brasileira de Cartografia, 69(1), 2017. Disponível em: $<$ http://www.seer.ufu.br/index.php/revistabrasileiracartografia/article/view/44040>. Acesso: 19 de junho de 2018.

ASSIS DIAS, M. C.; SAITO, S. M.; DOS SANTOS ALVALÁ, R. C.; STENNER, C.; PINHO, G.; NOBRE, C. A.; LIMA, C. O. Estimation of exposed population to landslides and floods risk areas in Brazil, on an intra-urban scale. International Journal of Disaster Risk Reduction, 31, 449-459. 2018. https://doi.org/10.1016/j.ijdrr.2018.06.002

AVILA, M. R. R.; MATTEDI, M. A. Desastre e território: a produção da vulnerabilidade a desastres na cidade de Blumenau/SC. Revista Brasileira de Gestão Urbana, v. 9, n. 2, 2017. http://dx.doi.org/10.1590/2175-3369.009.002.ao03

BLAIKIE, P.; CANNON, T.; DAVIS, I.; WISNER, B. At risk: natural hazards, people's vulnerability and disasters. Routledge. 2004

BRASIL. Lei 12796. Altera a Lei no 9.394, de 20 de dezembro de 1996, que estabelece as diretrizes e bases da educação nacional, para dispor sobre a formação dos profissionais da educação e dar outras providências. Brasília: Presidência da República, 2013. Disponível em: $\quad<$ http://www.planalto.gov.br/ccivil_03/_Ato2011-2014/2013/Lei/L12796.htm\#art1, 2013>. Acesso: 14 de junho de 2016.

BRASIL. Lei $\mathbf{n}^{\circ} \mathbf{1 0 . 7 4 1}$, de $1^{\circ}$ de outubro de 2003. Dispõe sobre o Estatuto do Idoso e dá outras providências. Diário Oficial da União. 2003. Disponível em: $<$ http://www.planalto.gov.br/ccivil_03/leis/2003/110.741.htm>. Acesso: 11 de outubro de 2018.

BRASIL. Ministério da Integração Nacional. Publicações: COBRADE: codificação, classificação, definição e simbologia dos desastres. Disponível em: $<$ http://www.mi.gov.br/publicacoes-sedec $>$. Acesso: 28 de agosto de 2017.

CARDOSO, A. L. Risco urbano e moradia: a construção social do risco em uma favela do Rio de Janeiro. Cadernos Ippur, v. 20, n. 1, p. 27-48, 2006.

CARMO, R. L. Urbanização e desastres: desafios para a segurança humana no Brasil. In: Carmo, R. L.; Valencio, N. (Org.). Segurança Humana em contextos de desastres. 1ed.São Carlos: Editora Rima, , p.1-14. 2014. Available at: $<$ https://www.nepo.unicamp.br/publicacoes/livros/segurancahumana/segurancahumana.pdf >. Acesso: 10 de novembro de 2018

CARVALHO, C. S.; MACEDO, E. S. D.; OGURA, A. T. Mapeamento de riscos em encostas e margem de rios. Brasília: Ministério das Cidades, 2007.

Cemaden (2019). Municípios monitorados. Disponível em: $<$ https://www.cemaden.gov.br/municipios-monitorados-2/>. Acesso: 10 de junho de 2019.

COSTA F. S. C.; PIMENTEL, M. A. Integrated Flood Risk Management And Local Communities In: Portugal And Brazil: Theoretical Contributions. Geograpphy Papers, 2017,63. http://dx.doi.org/10.6018/geografia/2017/294901

CPRM. Setorização de riscos. Disponível em: <http://www.cprm.gov.br/publique/GestaoTerritorial/Geologia-de-Engenharia-e-Riscos-Geologicos/Setorizacao-de-Riscos-Geologicos4138.html>. Acesso: 9 de junho de 2019.

CRED-UNISDR. (2015). The human cost of weather related disasters - 1995-2015. Disponível em: <https://www.unisdr.org/files/46796_cop21weatherdisastersreport2015.pdf>. Acesso: 16 de março de 2017.

CUTTER, S. L. A ciência da vulnerabilidade: modelos, métodos e indicadores. Revista Crítica de Ciências Sociais, n. 93, p. 59-69, 2011. https://doi.org/10.4000/rccs.165 
DEBORTOLI, N. S.; CAMARINHA, P. I. M.; MARENGO, J. A.; RODRIGUES, R. R. An index of Brazil's vulnerability to expected increases in natural flash flooding and landslide disasters in the context of climate change. Natural Hazards, 86(2), 557-582. 2017. https://doi.org/10.1007/s11069-016-2705-2

DESCHAMPS, M. Estudo sobre a vulnerabilidade socioambiental na Região Metropolitana de Curitiba. Cadernos Metrópole, 1(1), 191-219. 2008. https://doi.org/10.1590/8716

GOERL, R. F.; KOBIYAMA, M.; PELLERIN, J. R. G. M. Proposta metodológica para mapeamento de áreas de risco a inundação: estudo de caso do município de Rio Negrinho$\begin{array}{lllll}\text { SC. Boletim } & \text { de } & \text { Geografia, }\end{array}$ http://dx.doi.org/10.4025/bolgeogr.v30i1.13519

GONÇALVES, C. D. "Desastres naturais". Algumas considerações: vulnerabilidade, risco e resiliência. Territorium, [S.l.], n. 19, p. 5-14, dez. 2012. ISSN 1647-7723. Disponível em: $<$ https://impactum-journals.uc.pt/territorium/article/view/3067>. Acesso: 9 de junho de 2019.

HALLEGATTE, S.; VOGT-SCHILB, A.; BANGALORE, M.; ROZENBERG, J. Unbreakable: Building the Resilience of the Poor in the Face of Natural Disasters. Climate Change and Development. Washington, DC: World Bank. World Bank. 2017. Disponivel em: https://openknowledge.worldbank.org/handle/10986/25335,. Acesso em: 19 de novembro de 2018.

HUMMELL, B. M. L.; CUTTER, S. L.; EMRICH, C. T. Social vulnerability to natural hazards in Brazil. International Journal of Disaster Risk Science 7: 111, 2016. https://doi.org/10.1007/s13753-016-0090-9,

IBGE. Metodologia do censo demográfico 2010. Rio de Janeiro: IBGE. 720 p.ISBN 97885-240-4309-3.

$<$ https://biblioteca.ibge.gov.br/visualizacao/livros/liv95987.pdf $>$.; 2010. Acesso: 10 de julho de 2018.

IBGE (2010). Sinopse do censo demográfico. Disponível em: $<$ https://ww2.ibge.gov.br/censo2010/apps/sinopse/index.php?dados=10\&uf $=00>, \quad 2010$. Acesso: 10 de julho de 2018.

IBGE (2016) Base de Faces de Logradouros do Censo 2010. Disponível em: $<$ ftp://geoftp.ibge.gov.br/recortes para fins estatisticos/malha de setores censitarios/censo _2010/base_de_faces_de_logradouros > , 2016. Acesso: 10 de julho de 2018.

IBGE; CEMADEN (2018). População em áreas de risco no Brasil. Disponível em: $<$ https://www.ibge.gov.br/geociencias-novoportal/organizacao-do-territorio/tipologias-doterritorio/21538-populacao-em-areas-de-risco-no-brasil.html?=\&t=acesso-ao-produto $>$. Acesso: 15 de septembro de 2018.

JACOBI P. R.; MOMM-SCHULT, S. I.; BOHN, N. Acción y reacción. Intervenciones urbanas y el papel de las instituciones en post-desastre en Blumenau (Brasil). Revista EURE-Revista de Estudios Urbano Regionales. 2013 Jan 2, 39(116). Disponível em: $<$ https://www.eure.cl/index.php/eure/article/view/227/576>. Acesso: 10 de abril de 2017.

LIU, X.; YUE, Z. Q.; THAM, L. G.; LEE, C. F. Empirical assessment of debris flow risk on a regional scale in Yunnan Province, southwestern China. Environmental Management, 30(2), 249-264, 2002. https://doi.org/10.1007/s00267-001-2658-3

LONDE, L. R.; MOURA, L. G.; COUTINHO, M. P.; MARCHEZINI, V.; SORIANO, E. (2018). Vulnerabilização, Saúde E Desastres Socioambientais No Litoral De São Paulo: Desafios Para O Desenvolvimento Sustentável. Ambiente \& Sociedade, 21, e01022. Epub 08 de novembro de 2018.https://dx.doi.org/10.1590/1809-4422asoc0102r2vu1811ao

MARANDOLA JR., E.; HOGAN, D. J. Vulnerabilidades e riscos: entre geografia e demografia. Revista Brasileira de Estudos de População, 22(1), 29-53. 2005. DOI: http://dx.doi.org/10.1590/S0102-30982005000200009

MARANDOLA JR., E.; DANTONA, A. O. Vulnerabilidade: problematizando e operacionalizando o conceito. Segurança humana no contexto dos desastres. 1ed São Carlos: RiMa, p. 45-61, 2014. 
MARCHEZINI, V; WISNER, B. Challenges for vulnerability reduction in Brazil: insights from the PAR framework. In: MARCHEZINI, V. et al. Challenges for vulnerability reduction in Brazil: insights from the PAR framework. Reduction of vulnerability to disasters: from knowledge to action. São Carlos: Rima, p. 57-96, 2017.

MAZURANA, D.; BENELLI, P.; WALKER, P. How sex-and age-disaggregated data and gender and generational analyses can improve humanitarian response. Disasters, 37, S68S82. 2013. DOI: https://doi.org/10.1111/disa.12013

MENEZES, J. A.; CONFALONIERI, U.; MADUREIRA, A. P.; DE BRITO DUVAL, I.; DOS SANTOS, R. B.; MARGONARI, C. Mapping human vulnerability to climate change in the Brazilian Amazon: The construction of a municipal vulnerability index. PloS One, 13(2), e0190808, 2018. https://doi.org/10.1371/journal.pone.0190808

MIRANDOLA, F. A.; DE MACEDO, E. S. Proposta de classificação do Tecnógeno para uso no mapeamento de áreas de risco de deslizamento. Quaternary and Environmental Geosciences, 5(1), 2014. https://doi.org/10.5380/abequa.v5i1.34764

NEUMAYER, E.; PLÜMPER, T. The gendered nature of natural disasters: The impact of catastrophic events on the gender gap in life expectancy, 1981-2002. Annals of the Association of American Geographers, 97(3), 551-566. 2007. https://doi.org 10.1111/j.1467-8306.2007.00563.x

RONCANCIO, D. J.; NARDOCCI, A. C. Social vulnerability to natural hazards in São Paulo, Brazil. Natural Hazards, 84(2), 1367-1383, 2016. https://doi.org/10.1007/s11069016-2491-x

SAITO, S. M. Vulnerabilidades No Contexto De Sistemas De Alerta De Risco De Desastres. Revista Gestão \& Sustentabilidade Ambiental 7: 618-630, 2018.

SAMPAIO, T. Q.; PIMENTEL, J.; SILVA, C. R.; MOREIRA, H. F. A atuação do Serviço Geológico Do Brasil (CPRM) na gestão de riscos e resposta a desastres naturais. In: Anais.. VI Congresso de Gestão Pública - CONSAD. Brasília, 16 a 18 de abril de 2013. Disponível em: <http://consad.org.br/vi-congresso-consad-trabalhos-apresentados/>. Acesso: 9 de junho de 2019.

SHADECK, R.; SANTOS, M. S.; SCHNORR, T. M.; PEIXOTO FILHO, G. E. A atuação da secretaria nacional de defesa civil (Sedec) na gestão de riscos e resposta a desastres naturais In: Anais VI Congresso de Gestão Pública - CONSAD. Brasília, 16 a 18 de abril de 2013. Disponível em: <http://consad.org.br/vi-congresso-consad-trabalhosapresentados/>. Acesso: 9 de junho de 2019.

TORRES, H. D. G. A demografia do risco ambiental. População e meio ambiente: debates e desafios. São Paulo: Senac, 53-73. 2000

UN (2006). Conference Of European Statisticians: Recommendations For The 2010

Censuses Of Population And Housing. 213p. Disponível em:

$<$ http://www.unece.org/fileadmin/DAM/stats/publications/CES 2010 Census Recommendat ions_English.pdf $>$.

UN (2015). Department of Economic and Social Affairs. Principles and Recommendations for Population and Housing Censuses. 316p. Disponível em: $<$ https://unstats.un.org/unsd/demographic-social/Standards-and-

Methods/files/Principles and Recommendations/Population-and-Housing-

Censuses/Series M67rev3-E.pdf $>$.

UNISDR, United Nations International Strategy for Disaster Reduction. (2015). Sendai framework for disaster risk reduction 2015- 2030. Disponível em: $<$ http://www.wcdrr.org/uploads/Sendai Framework for Disaster Risk Reduction 20152030.pdf>. Acesso: 19 de Agosto de 2018.

WAMSLER, C.; BRINK, E.; RENTALA, O. Climate change, adaptation, and formal education: the role of schooling for increasing societies' adaptive capacities in El Salvador and Brazil. Ecology and Society, 17(2), 2012. http://dx.doi.org/10.5751/ES-04645-170202

ZHANG, N.; HUANG, H.; SU, B.; ZHANG, H. Population evacuation analysis: considering dynamic population vulnerability distribution and disaster information dissemination. Natural Hazards, 69(3), 1629-1646, 2013. http://dx.doi.org/10.1007/s11069-013-0767-y 